\title{
Espacio e inteligencia en Arturo Ardao: su contribución a una filosofía de la cultura americana
}

\author{
Space and intelligence in Arturo Ardao: his contribution to an \\ American philosophy of culture
}

Fernando Martin-De Blassi*

\begin{abstract}
Resumen: Este trabajo aborda la comprensión que A. Ardao desarrolla sobre el espacio y su primacía con respecto al tiempo. En otros términos, el tiempo como subsidiario del espacio, así como la razón en cuanto subsidiaria de la inteligencia. Esta misma inversión categorial fundamenta la ocupación que nuestro autor asigna al ejercicio del filosofar latinoamericano como una tarea afincada en una geografía concreta, delimitada por parámetros culturales y protagonizada por un sujeto que no es una sustancia racional, sino un ente biofísico en quien se clausura la historia como geo-historia y se da paso al desarrollo de una astro-historia a partir de la conquista del espacio exterior.
\end{abstract}

Palabras clave: Arturo Ardao, espacio, inteligencia, pensamiento latinoamericano.

\begin{abstract}
This work addresses the understanding that A. Ardao develops about space and its primacy with respect to time. In other words, time as a subsidiary of space, as well as reason as a subsidiary of intelligence. This same categorical investment bases the occupation that our author assigns to the exercise of the Latin American philosopher as a task based on a specific geography, delimited by cultural parameters and starring a subject that is not a rational substance, but a biophysical entity in which the history as geo-history closes, giving way to the development of an astro-history from the conquest of outer space.
\end{abstract}

Key words: Arturo Ardao, space, intelligence, Latin American thought

\footnotetext{
* Profesor y Licenciado en Filosofía por la Facultad de Filosofía y Letras de la UNCuyo, Argentina, becario doctoral del Consejo Nacional de Investigaciones Científicas y Técnicas (CONICET). Ha sido becario de posgrado durante un año por la Confederación suiza en la Université de Fribourg y se desempeña actualmente como docente de Filosofía de la Historia en la Facultad de Filosofía y Letras de la Universidad Nacional de Cuyo. Dirección electrónica: martindeblassi@hotmail.com
} 


\section{Presentación}

El pensador uruguayo Arturo Ardao expone una filosofía pluralista donde se advierte una diversidad de factores históricos, culturales y económicos. Esto implica que tal análisis no se reduce a la interpretación de una narrativa única que presenta los factores mencionados desde una cosmovisión lineal de la historia a partir de categorías tales como las de sucesión y simultaneidad, por no decir de progreso. Formula, por lo demás, una crítica a la noción antropomórfica de causalidad que ha sido planteada a partir de la inversión copernicana y, luego, de la teoría de Einstein acerca de la relatividad. Asimismo, el pensamiento filosófico de Ardao coincide en algunos conceptos fundamentales con dos autores que serán objeto de su análisis: J. E. Rodó y C. Vaz Ferreira. Con el primero concuerda en la concepción de que la realidad es cambiante en términos de una ontología del devenir y de la diferencia; ignorada ciertamente por la ontología tradicional que concibe al sujeto como una sustancia supra-temporal cuya universalidad desconoce la historia que construye. El libro Espacio e inteligencia, que comprende los ensayos de filosofía del espacio junto con sus ensayos de antropología, fue recopilado por Ardao en Venezuela en 1983 y reeditado en Montevideo diez años más tarde. Tales ensayos pertenecen a las décadas del '60 y del '70. En ellos se afirma, entre otros presupuestos, que la inteligencia es inclusiva de la razón así como el espacio es inclusivo del tiempo, lo cual implica el descentramiento de la geo-historia como narrativa propia de una época en particular, a saber, la que legitima el discurso euro-céntrico. Esto trae como aparejado la apertura de otra visión filosófica del hombre y de la historia. ${ }^{1}$

Ardao se ha interesado también en lo que respecta a la función actual de la filosofía latinoamericana y reflexiona sobre tal aspecto a partir de las categorías sujeto-objeto, expresadas en términos de una filosofía americana o bien de una filosofía de lo americano. En ese sentido introduce el concepto de comunidad, dado que los sujetos que producen e intervienen activamente en la elaboración de una filosofía latinoamericana integran una comunidad histórica con una tradición de costumbres y cierta tonalidad cultural. Ello

1 Cfr. Tani, R., "La filosofía antropológica de Arturo Ardao: el puesto de la inteligencia en el cosmos", Cuyo, 20 (2003), pp. 38-39. 
permite relacionar el concepto de temporalidad que caracteriza la condición propiamente histórica de una comunidad, con el de espacialidad, una idea que incluye la exterioridad del cuerpo, así como las prácticas concretas que se realizan en esa realidad social. De esta manera, Ardao orienta la hermenéutica de textos cuya materialidad ha sido fijada por entes biofísicos en un espacio geográfico preciso. Este enfoque reivindica una perspectiva de análisis no académica, a partir del abordaje interdisciplinario y la búsqueda de una metodología propia en la promoción de una conciencia que permita conocer y expresar la identidad latinoamericana. ${ }^{2}$

En este trabajo se procurará incursionar en la comprensión que Ardao desarrolla sobre el espacio y su primacía con respecto al tiempo. En otros términos, el tiempo como subsidiario del espacio, así como la razón en cuanto subsidiaria de la inteligencia. Ello permitiría ingresar en el análisis de la función que nuestro autor asigna al ejercicio del filosofar latinoamericano como una tarea afincada en una geografía concreta, delimitada por parámetros culturales y protagonizada por un sujeto que no es una sustancia racional, sino un ente biofísico en quien se clausura la historia como geo-historia y se da paso al desarrollo de una astro-historia a partir de la conquista del espacio exterior.

\section{Síntesis biográfica y doctrinal}

Nuestro pensador, abogado, historiador, escritor y periodista ha contribuido al estudio de la Historia de las ideas, en especial, de las ideas filosóficas en América Latina. De acuerdo con algunas apreciaciones de J. C. Torchia Estrada, Ardao es uno de los más importantes historiadores del pensamiento filosófico latinoamericano. Ha trazado el panorama completo de la historia de las ideas en el Uruguay y, dentro de ese panorama, ha hecho contribuciones fundamentales a varias de esas figuras, entre ellas Rodó y Vaz Ferreira. Asimismo, extendió su labor de historiografía filosófica a otros pensadores y corrientes de América Latina e, incluso, de España; cabe mencionar, aunque solamente a modo de ejemplo, un estudio suyo sobre la

${ }^{2}$ Cfr. Ibidem, pp. 39-42. 
filosofía de A. Bello y dos obras sobre B. J. Feijóo. ${ }^{3}$ Asimismo, realizó las investigaciones más minuciosas que se conocen sobre el origen del nombre América Latina. Es por este motivo que se trata de un autor imprescindible para el tratamiento de la cuestión del origen, el sentido y la función de la filosofía latinoamericana. Por lo que a nuestro tema respecta, Ardao ha cultivado también desde una perspectiva teorética las relaciones e implicancias filosóficas que se dan entre el espacio y la inteligencia. En el orden generacional, ha sido testigo, intérprete y actor del proceso de intenso incremento que experimenta la filosofía latinoamericana y su historiografía a partir de la década de 1940. Esta época coincide, precisamente, con los comienzos de Ardao en su labor de escritor. En la medida en que contemporáneos suyos, cercanos en el trato o la estimación, permiten trazar un perfil de época, podría decirse que la figura de Ardao estuvo relacionada con la de F. Romero, J. Gaos, P. Henríquez Ureña y L. Zea; este último, compañero entrañable de generación. ${ }^{4}$

Nuestro autor nació en Lavalleja, Uruguay, el 27 de septiembre de 1912 y falleció en Montevideo, el 22 de septiembre de 2003. Doctor en Derecho y Ciencias Sociales por la Universidad de la República de su país natal. Vinculado a esa casa de estudios, entre 1949 y 1974 fue profesor de Historia de las Ideas en América en la Facultad de Humanidades y Ciencias. De esa Facultad de Humanidades desempeñó los cargos de Decano (1968-1972) y Director del Instituto de Filosofía (1963-1974). Su primera obra, publicada en 1937, se llamó Vida de Basilio Muñoz, co-autor de este libro fue J. Castro y su

${ }^{3}$ Entre sus obras publicadas más destacadas, se encuentran: Vida de Basilio Muñoz (en coautoría con Julio Castro, Montevideo, 1937); Filosofía pre-universitaria en el Uruguay (Montevideo, 1945); Espiritualismo y positivismo (México, 1950); Batlle y Ordónez y el positivismo filosófico (Número, Montevideo 1951); La filosofía del Uruguay del siglo XX (México, 1956); Racionalismo y Liberalismo en el Uruguay (Montevideo, 1962); La filosofía polémica de Feijóo (Buenos Aires, 1962); Filosofía en lengua española (Montevideo, 1963); Etapas de la inteligencia uruguaya (Montevideo, 1971); Espacio e Inteligencia (Caracas, 1976); Génesis de la idea y el nombre de América Latina (Caracas, 1980); La inteligencia latinoamericana (Montevideo, 1991); España en el origen del nombre América Latina (Montevideo, 1992); Lógica y metafísica en Feijóo (Montevideo, 1997); y La lógica de la razón y la lógica de la inteligencia (Montevideo, 2000).

${ }^{4}$ Cfr. Torchia Estrada, J. C., "Arturo Ardao (1912)", en Clara A. Jalif de Bertranou (comp.), Semillas en el tiempo, Mendoza, EDIUNC, 2001, pp. 17-18. 
prólogo estuvo escrito por C. Quijano. Con ambas personalidades, Ardao participó en 1939 de la fundación del semanario Marcha. Ante la Asamblea de la Unesco celebrada en París en 1958, integró la delegación de Uruguay, formando parte asimismo, en 1967, de la Delegación de la Universidad de la República por Convenio con la Unesco en París. Durante el período 19761988 enseñó en la Universidad Simón Bolívar de Caracas, donde permaneció exiliado a raíz de la llegada de la dictadura militar a Uruguay. Participó igualmente como investigador del Centro de Estudios Latinoamericanos Rómulo Gallegos. Además de premios nacionales de alta distinción, obtuvo el Premio Interamericano Gabriela Mistral (1991) y el Morosoli de Oro (1995) que otorga la fundación Lolita Ruibal. La Universidad de la República le otorgó en 1992 el Doctorado Honoris Causa. ${ }^{5}$

La labor de Ardao como periodista comenzó en 1931 durante los últimos meses del diario El Nacional y la fundación inmediata del semanario Acción en 1932. En 1939, este último periódico se transforma en Marcha y su actividad de difusión continuará a través de la edición del mensuario Cuadernos de Marcha. Cabe señalar que en la vida del pensador uruguayo este tipo de actividad ha tenido siempre desde sus comienzos un lado cultural, primero como anticipo y luego como extensión del trabajo académico. En su ejercicio periodístico, Ardao se enfrentó a dos dictaduras en 1933 y 1973, respectivamente. Entre una y otra, por cierto, hubo diferencias en función del contexto nacional e internacional en el que se desarrollaron; si bien ambas se realizaron sobre la base de un mismo denominador común: el avasallamiento de las instituciones democráticas y de los derechos humanos durante el curso de una década. En este sentido, la prensa constituyó para Ardao un medio no sólo para expresar sus ideas, sino también para ejercer su deber cívico como ciudadano libre, partidariamente independiente. A pesar de su aguda conciencia de los cambios que se producen en una realidad social, nacional y cultural, Ardao manifiesta que Uruguay no ha claudicado en su razón de ser como país y en su irrevocable destino democrático, asentado por Artigas. ${ }^{6}$

Con respecto a la denominación de América Latina, Arturo Ardao manifiesta que ese nombre reviste una importancia en los órdenes tanto

5 Esta sucinta reseña biográfica se encuentra online en el sitio http://es.wikipedia.org/wiki/Arturo_Ardao

${ }^{6}$ Cfr. Loustaunau, F., "Entrevista a Arturo Ardao", Cuyo, 20 (2003), pp. 117-119. 
político cuanto económico y cultural. Su designación fue una preocupación constante en los patriotas independentistas. Aun cuando a mediados del siglo XIX el nombre de América Latina había sido propagado por Torres Caicedo, se impuso gracias a Martí y Rodó desde fines del siglo XIX y principios del siglo XX. Pero es a partir de la segunda mitad del siglo XX que este nombre se consagra de modo universal, sin por ello afectar la legitimidad de los nombres de Hispanoamérica e Iberoamérica.

En cuanto al término filosofía uruguaya, para Ardao hace referencia a la que ha sido cultivada por los uruguayos desde las circunstancias locales. Esto ha existido desde el comienzo académico a fines del siglo XVIII en tanto que conciencia filosófica de la incipiente inteligencia nacional. Paulatinamente, fue adaptando y adoptando el pensamiento europeo a la circunstancia propiamente uruguaya y, finalmente, con Vaz Ferreira pasó de ser filosofía en el Uruguay a filosofía del Uruguay, es decir, en sentido estrictamente creador o productor. Afirmaciones análogas propondrá nuestro pensador acerca del estatuto de la filosofía latinoamericana y su función actual, tema que será abordado en el último apartado de este trabajo. ${ }^{7}$

\section{Una nueva comprensión del espacio, de la inteligencia y de la condición antropológica}

Ha sido mencionado que Espacio e inteligencia es un texto editado en 1983 en Caracas, ciudad del exilio de Ardao, y recoge artículos producidos entre los años 1963 y 1984. Muestran el interés de nuestro autor por un abordaje explícitamente filosófico acerca de una nueva interpretación de la historia y la antropología, en la cual los conceptos de espacio e inteligencia ocupan un lugar central.

En "Relaciones entre el espacio y la inteligencia" (1976), nuestro autor considera que la noción de espacio vivido debe comprender la del espacio del mundo circundante, del espacio intencional de la conciencia y del espacio vital del organismo, tanto biológico como social. El pensador uruguayo manifiesta que Bergson denomina a la duración tiempo vivido, cualitativo y concreto,

${ }^{7}$ Cfr. Ibidem, pp. 114-117. 
heterogéneo, en contraste con la extensión, es decir, el espacio contrapuesto a la vida, cuantitativo y abstracto, homogéneo. Sin embargo, lo que en verdad vivimos inseparablemente -aduce Ardao- es el espacio-tiempo uno. La medida cuantitativa que se utiliza para formalizar el despliegue temporal es simbólica, distante del tiempo real, así como las convenciones geométricas que mensuran el espacio no se identifican con el espacio real. Pero ello no quiere decir que sean símbolos ilusorios.

En otro orden de ideas, Ardao expresa que es preciso llevar a cabo una distinción entre razón e inteligencia. La primera considera que el tiempo se manifiesta en algún lugar recóndito de la realidad con independencia del espacio. La segunda, empero, capta el movimiento del tiempo porque lo ha hecho primero desde un determinado espacio. En cuanto facultades, la razón es analítica, es decir, relaciona, identifica y cuantifica; la inteligencia, por su parte, es capacidad aprehensora de totalidad, capta la diversidad y la cualidad antitéticas de la identidad y la cantidad.

De acuerdo con lo dicho, la inteligencia, entonces, aprehende el tiempo real porque primero comienza aprehendiendo el espacio desde el cual capta ese tiempo. Esto quiere decir que la inteligencia es utilizada por un sujeto que tiene conciencia del espacio para la representación simbólica del tiempo. Por ser una cualidad de ese sujeto, ella establece la posibilidad de toda síntesis. El recurso metodológico al espacio no es artificial sino natural, pues en su pureza natural el tiempo es espacio. El individuo piensa el tiempo en una línea recta y esa línea recta contiene series espaciales que se entienden como días, semanas, meses y años. Por medio del ejemplo del reloj de péndulo, Ardao explica que el movimiento del péndulo se desplaza de un extremo a otro recorriendo una distancia espacial, lo que nos ayudaría a entender que el tiempo no se concibe abstraído de cualquier condición espacial. Por el contrario, la noción de tiempo se vale de elementos materiales y espaciales para su construcción. Existe, por ello, una relación entre tiempo real y espacio real que no se podría considerar como ilusorio.

De esta manera, nuestro autor sostiene que es imposible llegar a concebir un tiempo purgado de toda espacialidad, como si fuese un concepto abstracto y puro. Tiempo y espacio son una unidad necesaria, de manera tal que, para concebir el tiempo, se precisa la manifestación previa del espacio. El tiempo es el espacio punto por punto, la oceánica masa fluida del espacio, 
es el cuándo dependiendo del dónde. Toda idea de la temporalidad de la existencia humana conduce directamente a la de la edad personal. El sujeto, al estar dotado de edad, se encuentra inserto en los procesos de la tierra, formando parte de ellos. Él mismo es consciente a la vez de poseer memoria que le permite dirigirse a sus recuerdos temporales utilizando como base las vivencias, el espacio vivido. ${ }^{8}$

Sobre la base del planteo anterior, en "La antropología filosófica y la espacialidad de la psique" (1963), Ardao prosigue con el estudio de una antropología filosófica que explicite las nociones de espacio-tiempo en relación con el ser humano y su estadio de conciencia. A su juicio, la antropología hasta el momento estudiada es una propuesta que se basa en el modelo antropológico kantiano o de cuño positivista, modelo a partir del cual también termina trabajando Scheler, sin aportar algo distintivo al respecto. Si bien el ser humano no está escindido en vida animal y vida humana en el seno de lo biológico, la antítesis decisiva en estas formulaciones es la que separa a la vida (bio-psíquica) del espíritu. Lo esencial del hombre es el espíritu que es supra-material y supra-temporal. Así, entonces, los planteos posteriores van a suponer la existencia del espíritu y la psique de un modo atemporal, mientras que asociarán lo orgánico a lo espacial y temporal. Aquí se instala el problema de la dualidad antropológica cartesiana, en la medida en que la escisión mentada entre res cogitans y res extensa concluye en la afirmación de la no espacialidad de la psique y el espíritu como planos yuxtapuestos a lo tempoespacial de lo orgánico.

Ardao observará que el prejuicio dualista y antropocéntrico se afinca en una condición eminentemente temporal de la conciencia que niega su misma condición espacial. Esta tesis alude principalmente a la falacia temporalista, según la cual el modo de razonar se manifiesta en los fenómenos psíquicos con independencia del espacio y del cuerpo en el cual acontecen. Se trata de un prejuicio recurrente en la historia de la filosofía admitir o postular que la razón, como fenómeno psíquico (espiritual), se realiza en forma independiente del cuerpo y del espacio social e histórico. Por el contrario, el concepto espacial de la inteligencia en Ardao se relaciona directamente con el

8 Cfr. Ardao, A., "Relaciones entre el espacio y la inteligencia", en su Espacio e inteligencia, $2^{\mathrm{a}}$ ed., Montevideo, Fundación de Cultura Universitaria/Biblioteca de Marcha, 1993, pp. 13-36. 
concepto de creatividad y su relación con lo humano, al modo como lo psíquico se relaciona con lo orgánico. La inteligencia creativa integra la razón, los sentimientos, la imaginación y los instintos. Se trata de entender que los procesos afectivos, volitivos y cognitivos se producen en un cuerpo vivo, que es organismo espacial, un volumen que ocupa lugar en el espacio. Se integran, en este sentido, las circunstancias históricas, existenciales, éticas y socioculturales que permiten comprender al individuo como situado en el espacio. De allí que el concepto de sujeto deba ser pensado más allá del dualismo ontológico clásico, materialista o idealista. Ardao lo considera como un ente bio-físico pensante cuyas condiciones de existencia se desarrollan en el espacio-tiempo cosmológico. ${ }^{9}$ Dice:

La situación vital, psíquica, espiritual, histórica, socio-cultural, ética, es para el individuo, en primer término, situación en el espacio, conforme a la raíz etimológica del vocablo situación, situs, sitio, lugar. A partir de ahí, todas las demás connotaciones. Todo contenido de conciencia se localiza espacialmente, en el espacio inmanente al cuerpo y en el que trasciende a éste. Hay para lo psíquico un vivido aquí, cuyas determinaciones espaciales recorren el cuerpo desde los músculos y la piel, con la clave del rostro, a las vísceras y los centros, con la clave de la corteza cerebral; y otro aquí no menos vivido, que refiere al sitio planetario $-\mathrm{y}$ de ahora en adelante también eventualmente extraplanetario- donde el cuerpo se emplaza o se desplaza. ${ }^{10}$

\section{Crítica al concepto tradicional de causa: la nueva comprensión de praxis e historia}

En el ensayo "Sucesión y simultaneidad" (1987), Ardao critica la concepción clásica que ha interpretado la sucesión como causalidad, sin reparar en el concepto de simultaneidad. Dice que la temporalidad del espacio en cuanto extensión genera el orden de la simultaneidad; mientras que la

\footnotetext{
${ }^{9}$ Cfr. Ardao, A., "La antropología filosófica y la espacialidad de la psique”, en Espacio e inteligencia, pp. 37-54.

${ }^{10}$ Ibidem, p. 51.
} 
temporalidad del espacio intensivo genera el orden de la sucesión. En otras palabras, la espacialidad temporal extensa, que genera el concepto de simultaneidad, es exterioridad; mientras que la espacialidad temporal intensa, del orden de la sucesión, es interioridad. No se trata de la coexistencia de dos espacios, como si se tratase de una nueva forma de dualismo; sino, más bien, de un solo y mismo espacio, siempre temporal, que por un lado es exterioridad y por otro, interioridad. ${ }^{11}$

En "Crisis de la idea de historia como geo-historia" (1972), Ardao analiza distintas etapas de las concepciones filosóficas de la historia. Por un lado, se refiere a una línea de pensamiento propia del Iluminismo y su noción del progreso de la razón (Condorcet, Comte, Hegel, Humboldt), ajena a la tradición teológica de la religión sobrenatural; por otra parte, alude a otra línea con criterios diferentes que incluye ideas de la fe cristiana acerca del origen y la meta de la historia. Sin embargo, a pesar de esta profunda divergencia teofilosófica acerca de la historia, Ardao atestigua que ambas teorías son similares en relación con el desarrollo, planteamiento y conclusiones acerca del ángulo geo-histórico. A este planteamiento subyace la misma idea sustancial de unificación de la humanidad, del mundo y de la historia a través de un proceso en el que actúan como ejes el descubrimiento de América y la circunnavegación del globo. Todo ello, implica una clausura espacial definitiva de toda la historia proyectada sobre la corteza del planeta Tierra. Esta clausura, en la medida en que produce un descentramiento, cuestiona la narrativa teleológica que identificaba a la historia eurocéntrica como perspectiva de toda la humanidad. Por otra parte, Ardao propone que la meta de la historia debe replantearse desde una perspectiva astro-histórica puesto que el hombre ha comenzado en el siglo XX a proyectar y realizar viajes a otros planetas.

Tal crisis de la noción de historia como geo-historia se debe al hecho concreto que plantea la posibilidad de realizar la historia humana fuera del planeta Tierra. Si bien la tradición occidental ha fortalecido la idea de unificación de la humanidad en un sistema mundo, este proyecto globalizador se ha visto superado por los propios hechos que ha generado y que ponen en crisis la tesis etnocéntrica de tal proyecto, esto es, concebir, la historia como

${ }^{11}$ Cfr. Ardao, A., "Sucesión y simultaneidad", en Espacio e inteligencia, pp. 79-89. 
un devenir pensado indefectiblemente desde la Tierra. De esta manera, Ardao sostiene que, a partir de este descentramiento copernicano, a la geo-historia le sucede la así llamada astro-historia. Ello no implica un ensanche acumulativo, sino una radical contribución cualitativa, un novum que confiere una significación distinta al concepto mismo de historia universal. Como ya no se trata de fenómenos exclusivos del planeta Tierra, la puesta en marcha de la conquista del espacio exterior reclama una nueva consideración de la historia y la existencia humanas. ${ }^{12}$

La clave para interpretar este novum cualitativo se describe en "Praxis y espacio exterior" (1979). Allí, Ardao afirma que al realizar viajes por el exterior y conocer otros planetas, el ser humano es poseedor de una nueva perspectiva cognitiva y ontológica. Su filosofía plantea los problemas referidos a los conceptos de "mente", "materia", "vida" y "razón". Esta situación concreta presenta nuevos desafíos que obligan a descentrar la perspectiva o el foco de análisis de los debates clásicos en filosofía. En un horizonte de comprensión clásico, la metafísica defiende la distinción entre naturaleza y esencia asumiendo la esencia desde el plano de la inmanencia. La antropología filosófica, desde una perspectiva más bien científica, ha sustituido la distinción tradicional a favor de una concepción de raigambre evolucionista. Propone una ontología en proceso a partir de concepciones naturalistas, vitalistas y mecanicistas relacionadas con la biología. Se trata, empero, de concepciones dualistas que no conciben al ser humano como un ente biológico en tanto que ha comenzado a orientar la evolución a partir de descubrimientos como el ADN, la clonación de seres vivientes, el genoma humano, los trabajos de la inteligencia artificial y la neurociencia. Superando el dualismo metafísico de la antropología filosófica perteneciente a la tradición científica, estos procesos científico-tecnológicos revalorizan la división entre lo natural y lo artificial desde una nueva concepción de lo natural y lo humano. Tal forma de pensar las condiciones ontológicas en las que vivimos puede llegar, finalmente, a superar el concepto etnocéntrico de lo humano.

Ardao hace referencia también a una modalidad de trascendencia diferente de la propugnada por Bergson, cuya hipótesis argüía que el impulso vital de la conciencia supera el obstáculo que supone la materia. Para nuestro

${ }^{12}$ Cfr. Ardao, A., "Crisis de la idea de historia como geo-historia", en Espacio e inteligencia, pp. 91-106. 
pensador, empero, la materia no es obstáculo sino un medio de lograr la trascendencia misma hacia formas superiores de conciencia. La transformación cualitativa, que supone una posibilidad cierta de trascendencia de lo humano que supera todo pensamiento dualista, hará que el ente biofísico pensante asuma sus responsabilidades éticas y filosóficas en referencia a otras actividades auto-dirigidas que producirán nuevas transformaciones. ${ }^{13}$

\section{Inteligencia creadora y función del filosofar latinoamericano}

Ardao reivindica el valor de la inteligencia creadora, concreta y aplicada que resulta de la razón del sujeto histórico latinoamericano y, en su caso particular, uruguayo. Propone, en este sentido, desarrollar la inteligencia como actividad crítica creativa descentrada de la hegemonía de la razón al modo europeo. Esta filosofía entiende que la inteligencia creadora es capaz de pensar sus concreciones históricas como capacidad humana que se desarrolla en un espacio social, histórico y cosmológico. De allí la legitimidad de la pregunta por el estatuto de la filosofía latinoamericana no tanto como un ejercicio acerca de lo americano, sino como una elaboración crítica desde un sitio histórico específico.

Nuestro autor sostiene que, más allá de la universalidad (abstracta) que determinadas corrientes filosóficas pretenden atribuir al ejercicio filosófico, la filosofía es asunción de la historicidad humana. De esta manera, es preciso establecer una distinción previa para la delimitación de la función actual de la filosofía latinoamericana: ¿nos referimos a la filosofía como realidad ya fijada en el espíritu objetivo o bien a la filosofía como ejercicio todavía viviente en el espíritu subjetivo? Es decir, la distinción entre la filosofía como un determinado tipo de conocimiento históricamente acumulado, organizado y hasta institucionalizado, o bien la filosofía como tarea y misión del filósofo.

El obligado desdoblamiento del tema da lugar a dos afirmaciones: función actual del saber filosófico, función actual del pensar filosófico. El primero, apunta a la transmisión de la filosofía, es ante todo una cuestión pedagógica. El segundo afecta a la inteligencia latinoamericana en su

${ }^{13}$ Cfr. Ardao, A., "Praxis y espacio exterior”, en Espacio e inteligencia, pp. 107-121. 
responsabilidad por el ejercicio de la filosofía en lo que tiene de reflexión y especulación. Apela este último al compromiso de la inteligencia latinoamericana como entidad social, una entidad principalmente espacial que asume su historia cuyo presente se proyecta desde una determinada situación geográfica.

Cabe señalar que es recién en nuestro tiempo que ha comenzado a hablarse de función de la filosofía. El ascenso filosófico del concepto de función se vincula con el de estructura, que ha venido a desplazar al de sustancia. Toda función comporta el ejercicio de una actividad, y esa actividad lo es siempre de un elemento o miembro en relaciones de interdependencia dinámica, o interacción dialéctica, con los demás miembros del todo o conjunto del que forma parte. Es inseparable de la idea de conexión activa, que es propia de la de estructura, sea esta mecánica, biológica, psíquica o social. La filosofía es la que promueve el término "función" a un orden categorial y se ve ella misma afectada por tal cambio.

Esto quiere decir que la cuestión contemporánea de la filosofía, antes que en su objeto, reside en su función. Ya no es tematizada como un saber estático, quieto, que versa sobre determinadas esencias ellas mismas ahistóricas o ajenas al cambio. Se ha desplazado este punto de reflexión hacia la línea semántica que involucra al ejercicio de la filosofía como quehacer, tarea y misión en la medida en que esta misma se auto-comprende (autovalora) como un saber dinámico y en actividad. Lo que la filosofía es, sólo resulta considerable a partir de lo que ella es capaz de hacer y de cómo es capaz de hacerlo. Esta línea interpretativa ha sido elaborada a partir de conceptos tales como los de devenir, dialéctica, proceso, praxis y, fundamentalmente, historia. La categoría de historia refiere a una universalidad de la experiencia. Ha sido de una importancia inusitada en ella el ingreso de las conceptuaciones de conexión funcional, en el sentido de relación estructural. De esta manera, el saber histórico se convierte en un obligado recurso metodológico de la disciplina o forma del conocimiento que aspira a la universalidad mayor, noológica, que es la filosofía. Desde una lógica relacional, atenta a los elementos que van estableciendo las configuraciones de la experiencia social, la historia coloca a la filosofía, en tanto que función, en una óptica nueva. Es su condicionamiento socio histórico lo que aparece advertido. La filosofía, en este sentido, no puede menos que operar como 
fenómeno social, condicionado al mismo tiempo que condicionante. Siempre opera en acción recíproca o en interacción.

Ardao se ocupará en la función actual de la filosofía en Latinoamérica como aplicación del pensamiento filosófico a su radical destino de búsqueda, hallazgo y orientación. Esto supone un enjuiciamiento crítico. Dice que ese momento ha llegado a Latinoamérica y sólo la filosofía como ejercicio especulativo y crítico es, además de filosofía en Latinoamérica, filosofía latinoamericana.

Desde los orígenes coloniales hasta nuestros días ha habido filosofía en nuestra América, pero no siempre ha sido en rigor filosofía latinoamericana. Aunque desde los primeros tiempos también, fue surgiendo, desarrollándose y creciendo lo que sería como la parte dinámica de la filosofía en América Latina. No se ha tratado tan sólo de la filosofía recibida, acogida, transmitida o reproducida en función de centros ultramarinos de imperio político o cultural; sino también innovada y renovada por esfuerzos de adaptación y, eventualmente, de creación.

Su manifestación no representa el mero conocimiento filosófico, por funcional que en cualquier caso sea, sino el propio filosofar. En este segundo aspecto, el sujeto es productor del filosofar. Y esto se realiza en un cambio de sentido del ejercicio de la inteligencia en la medida en que el latinoamericano, sujeto del conocimiento filosófico, lo es cada vez en el carácter de sujeto del filosofar. Es por esta vía que, poco a poco, la filosofía latinoamericana ha quedado definitivamente constituida. Esto no significa que ella funcione en todas sus direcciones con autonomía espiritual. En el presente, en ciertos países, la filosofía latinoamericana ha alcanzado significativas expresiones de densidad y nivel. Se ha ido a planos más de creación, abandonando la adopción pasiva, adaptación reforzada por acción mecánica o refleja.

La estimación positiva de lo propio constituye su origen. Se asume Latinoamérica cada vez más reflexivamente en la peripecia de su historia, su cultura y sus gentes marginadas. Clarificándose a ese modo, es desde sus vitales circunstancias e intransferibles situaciones (subjetivas u objetivas) que se reconoce protagonista de la universalidad humana al igual que cualquier 
otra región del planeta, convocada a encarar con la misma libertad los más universales objetos filosóficos. ${ }^{14}$

${ }^{14}$ Cfr. Ardao, A., "Función actual de la filosofía en Latinoamérica", en La inteligencia latinoamericana, Montevideo, Universidad de la República, Dirección General de Extensión Universitaria, 1987, pp. 131-139. 